\title{
Symmetrical Unit-Cell Numerical Approach for Flip-Chip Underfill Flow Simulation
}

\author{
Fei Chong $\mathrm{Ng}^{1}$, Mohd Hafiz Zawawi ${ }^{2}$, Lun Hao Tung ${ }^{1}$, Mohamad Aizat Abas ${ }^{1,{ }^{*}}$, Mohd Zulkifly \\ Abdullah ${ }^{1}$
}

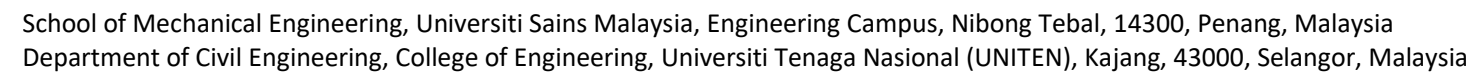

\section{Article history:}

Received 20 June 2020

Received in revised form 19 August 2020

Accepted 24 August 2020

Available online 30 August 2020

\begin{abstract}
Underfill process is a critical manufacturing process to safeguard and enhance the package reliability of flip-chip devices. In most underfill researches, the underfill flow was primarily investigated through numerical simulation. Nonetheless, the numerical simulation required a tremendously long time to complete. This paper presents a new symmetrical unit-cell approach to simulate the flip-chip underfill encapsulation process. The current numerical simulation is based on the finite volume method scheme. By exploiting the repetitive symmetry of bump array in flip-chip, the computational domain was simplified into a long array of unit-cells of one-pitch thick while the symmetrical walls between adjacent unit-cells were modelled using the periodic boundary condition. Accordingly, the computational costs can be greatly reduced. Alongside with the introduction of a new numerical approach of flip-chip underfill, the variation effect of bump pitch was studied by considering four flip-chip cases with bump pitches ranging from $0.08 \mathrm{~mm}$ to $0.16 \mathrm{~mm}$. The numerical findings were found to in great consensus to the referencing experimental data, with the discrepancy, not more than $14.54 \%$. Additional validation with the analytical filling time model revealed that both the numerical and analytical filling progressions are comparable. It is found that the increases in bump pitch can reduce the filling time at a particular filling distance, such that the filling time was halved within the investigated range of bump pitch. This new numerical approach is particularly useful for the simulation works of underfill process, especially the design and process optimization.
\end{abstract}

Keywords:

Bump pitch; Electronic packaging; Finite volume method (FVM); Flip-chip;

Underfill encapsulation

\section{Introduction}

Flip-chip packaging is a method to interconnect semiconductor device to the external circuitry through multiple array of solder bumps. The first flip-chip was introduced by IBM in the late 1960s [1]. In the electronic packaging, underfill encapsulation is a vital process to ensure the reliability and

\footnotetext{
* Corresponding author.

E-mail address: aizatabas@usm.my (Mohamad Aizat Abas)
}

https://doi.org/10.37934/cfdl.12.8.5563 
performance of flip-chip package. Figure 1 depicts the schematic of underfill encapsulation process. The underfill process is carried out during the manufacturing assemble line, by dispensing the underfill fluid into the small gap beneath the chip and substrate. The chip assembly is cured after the gap was filled with the underfill fluid, to yield a harden layer between the chip and substrate. The functions of underfill encapsulation include to relief the residual stress due to thermal mismatch, to serve as a protective layer and to promote the package's reliability [2-3]. There are various researches conducted to optimize the underfill process through package design [4-7] as well as to resolve the issues of incomplete filling [8] and void defect [7,9].

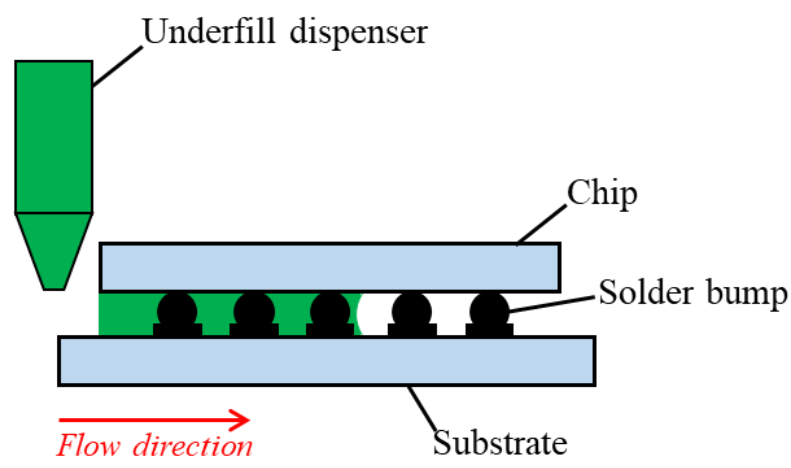

Fig. 1. Schematic of flip-chip underfill encapsulation process [10]

The most common and straightforward approach in underfill research is through experiment [2$3,5-11]$. However, the concerns of cost and limited versatility that arise from the underfill experiment prompt to the implementation of numerical simulation. There are variety of numerical schemes adopted in the underfill simulation, for instance, finite volume method (FVM) [6, 12-13], finite element method (FEM) [14], fluid/structure interaction (FSI) [8], discrete phase model (DPM) [10], lattice Boltzmann method (LBM) [7] and Petrov-Galerkin (PG) method [15]. As the underfill flow simulation usually based on the full flip-chip, therefore the numerical simulation took substantial long time to complete.

This paper herein presents a new approach for underfill simulation by considering the simplified symmetrical unit-cells array. The computational domain only consists of a long array of unit-cells arranged along the flow direction. In fact, the concept of unit-cell was pioneered by Yao et al., [16] in his work to determine numerically the permeability of flip-chip. Typically, a unit-cell consists of one solder bump confined in a square of sides equal to the bump pitch. The flip-chip bump array consists of unit-cells arranged in orderly quadrilateral manner. Due to the repetitive nature of bump array and the symmetry of the underfill flow front, it is sufficient to model the flip-chip domain as a long array of unit-cells with one-pitch thick along the flow direction. With the size reduction of computational domain, the associated mesh number will be reduced and ultimately achieve lower computational cost. To the best knowledge of authors, the long array of unit-cells coupled with periodic boundary condition was never adopted as the flow domain for the underfill flow simulation.

As the variation effect of bump pitch parameter regularly being studied in the past literatures [4$5,13]$, this paper also investigated the pitch variation on the filling time through the current introduced numerical approach. The current numerical variation work will be referencing the underfill experiment conducted by Lee et al., [5] whom utilized four glass flip-chips with bump pitches vary from $0.08 \mathrm{~mm}$ to $0.16 \mathrm{~mm}$ and the glycerin as experimental underfill fluid. To date, the variation study of bump pitch scarcely being investigated numerically. 


\section{Numerical Simulation}

The three-dimensional capillary underfill fluid flow in flip-chip encapsulation process was numerically simulated using the commercially available finite volume method (FVM) based software, ANSYS Fluent. The underfill fluid flow is modelled to be unsteady, laminar and incompressible [6, 1213]. The governing equations to be solved through discretization of FVM scheme are continuity equation and Navier-Stokes equation, respectively given as follows:

$$
\begin{aligned}
& \frac{\partial \rho}{\partial t}+\nabla \cdot(\rho \overrightarrow{\mathrm{u}})=0, \\
& \frac{\partial}{\partial t}(\rho \overrightarrow{\mathrm{u}})+\nabla \cdot(\rho \overrightarrow{\mathrm{u}} \cdot \overrightarrow{\mathrm{u}})=-\nabla p+\nabla \cdot \overline{\bar{\tau}}+\rho \overrightarrow{\mathrm{g}},
\end{aligned}
$$

with density, $\rho$, velocity, $\overrightarrow{\mathrm{u}}$, pressure, $p$, shear stress tensor, $\overline{\bar{\tau}}$ and gravity, $\overrightarrow{\mathrm{g}}$. The implicit multiphase volume of fluid (VOF) model is set to track the flow front of the underfill fluid, which governed by the transport equation below:

$$
\frac{\partial f_{n}}{\partial t}+\vec{u} \cdot \nabla f_{n}=0
$$

There are two distinct fluid phases in the current simulation, such that the primary phase is air while the secondary phase is the underfill fluid. The material properties of both phases used in the simulation were given in Table 1.

Table 1
\begin{tabular}{lll} 
Material properties of primary phase and secondary phase \\
\hline Material properties & Primary phase: Air & Secondary phase: Glycerin [5] \\
\hline Density $\left(\mathrm{kg} / \mathrm{m}^{3}\right)$ & 1.184 & 1232 \\
Viscosity $(\mathrm{Pa}-\mathrm{s})$ & $1.85 \times 10^{-5}$ & 1.5 \\
Surface tension $(\mathrm{N} / \mathrm{m})$ & - & 0.064 \\
Contact angle $\left(^{\circ}\right)$ & - & 55 \\
\hline
\end{tabular}

Generally, a flip-chip can be characterized based on three geometrical parameters: bump pitch, $W$, bump diameter, $d$ and gap height, $h$, as shown in Figure 2. For the current simulation works that based on the past underfill experiment [5], there are four cases of flip-chips with varying bump pitch to be considered, $W=0.08 \mathrm{~mm}, 0.10 \mathrm{~mm}, 0.12 \mathrm{~mm}$ and $0.16 \mathrm{~mm}$. However, both the bump diameter and gap height are constant for all flip-chips and having a similar value of $0.05 \mathrm{~mm}$. As the flip-chips investigated consist of repetitive bump arrays arranged in quadrilateral manner as shown in Figure 2 , simplification can be made on the mathematical fluid domain to reduce the both the complexity and thus the mesh number of the mathematical model. Therefore, the computational costs can be greatly cut while preserving the accuracy of numerical results. As the result of exploiting the symmetry of flip-chip bump array, the effective fluid domain is a long array of unit cells of one-pitch thick along the flow direction, as depicted in Figure 3(a). The walls of the cut-planes between adjacent unit-cells were modelled as the periodic boundary condition. Both pre-bump and post-bump length are equals to $0.2 \mathrm{~mm}$, while the number of bump rows are dependent on the pitch. The number of bump rows is chosen such that the total length of flow domain is at least $2.5 \mathrm{~mm}$, such that to cover the investigated filing distance of $2.0 \mathrm{~mm}$. The boundary conditions applied on the fluid domain consists of pressure inlet, pressure outlet, periodic and no-slip wall, as shown in Figure 3(b). 


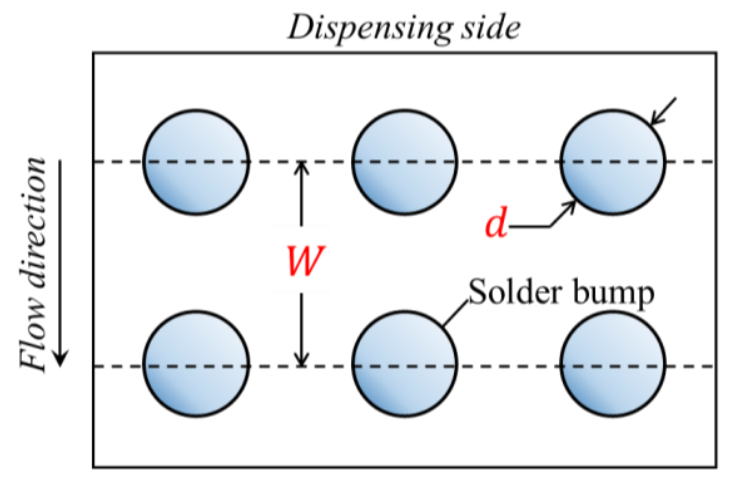

(a) Top view

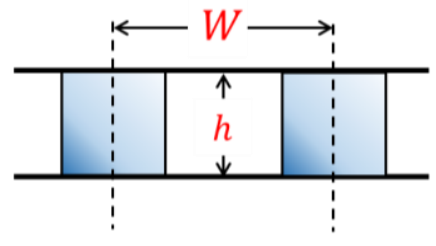

(b) Side view

Fig. 2. Geometrical representation of solder bump array in a flip-chip [13]

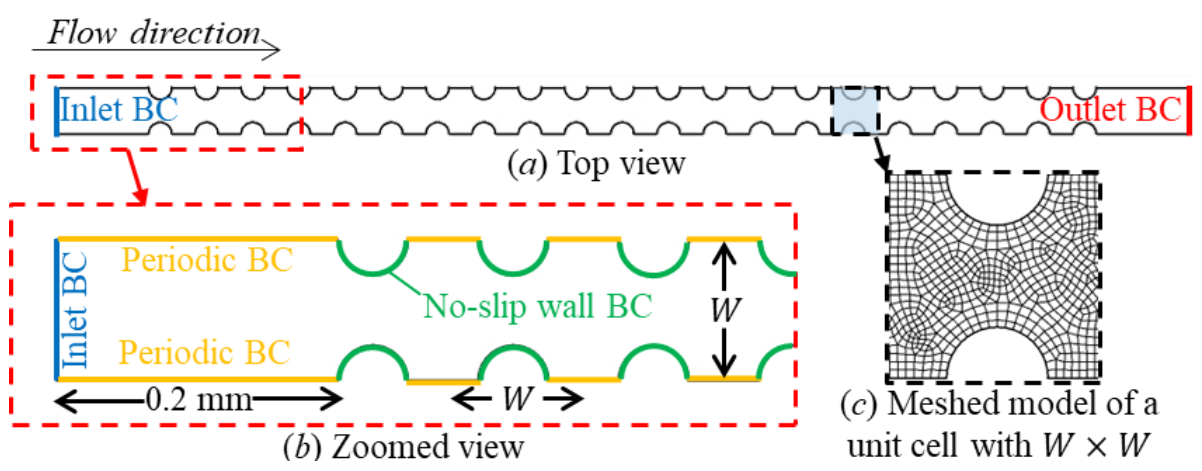

Fig. 3. Overview of symmetrical unit cells numerical model

Structural hexagonal mesh is applied on the mathematical model, as shown in Figure 3(c). To determine the optimum mesh sizing to be used on the model, a mesh independent study based on the filling times is conducted as shown in Table 2. It is found that the discretization error drops below $5 \%$ beyond mesh model III, such that when the mesh element number exceeded 86,130. Upon additional considering the computational time, the mesh sizing and setting from the mesh model $\mathrm{V}$ is applied for all four cases of current numerical models.

\section{Table 2}

Mesh independent study for long array unit-cells

\begin{tabular}{llll}
\hline $\begin{array}{l}\text { Mesh } \\
\text { model }\end{array}$ & $\begin{array}{l}\text { Element } \\
\text { number }\end{array}$ & $\begin{array}{l}\text { Filing times } \\
\text { (s) }\end{array}$ & $\begin{array}{l}\text { Discretization } \\
\text { error }\end{array}$ \\
\hline I & 46,856 & 1.27013 & $15.25 \%$ \\
II & 61,488 & 1.26073 & $15.87 \%$ \\
III & 86,130 & 1.47124 & $1.83 \%$ \\
IV & 110,660 & 1.45220 & $3.10 \%$ \\
V & 176,436 & 1.49861 & Ref. \\
\hline
\end{tabular}

This simulation work adopted the pressure-based numerical solver; while the SIMPLE and second order upwind algorithm schemes were used respectively for pressure-velocity coupling and spatial discretization. Moreover, the variable time stepping with unity global Courant number is selected, with the time steps ranging from $10^{-5} \mathrm{~s}$ to $10^{-2} \mathrm{~s}$. The default setting on iterative solver was used where the numerical model will be solved iteratively until the convergence achieved with residual error smaller than the order of $10^{-6}[6]$. Finally, the simulations were executed with the parallel solver of 7 process. The specifications of computer are Intel XEON $3.70 \mathrm{GHz}$ octa-cores CPU and 16 GB RAM. 


\section{Results and Discussion}

\subsection{Validation with Past Underfill Experiment}

To affirm the accuracy of current numerical models, a quantitative validation was conducted with the reference of past underfill experiment [5], for which the current numerical simulation was based on. Both the experimental and numerical filling times for the flow front to travel a distance of $2 \mathrm{~mm}$ from the inlet are presented in Table 3. It is found that the both the simulation and experiment achieved great consensus, as the discrepancy observed is not more than $14.54 \%$. On the trend, both numerical simulation and experiment concluded that the filling time for the underfill fluid a fixed distance decreases with the increases in bump pitch.

Table 3

Comparison of experimental [5] and numerical filling times in various pitch cases

\begin{tabular}{llll}
\hline $\begin{array}{l}\text { Bump pitch, } \\
W(\mathrm{~mm})\end{array}$ & $\begin{array}{l}\text { Experimental filling time } \\
(\mathrm{s})\end{array}$ & Current numerical filling time $(\mathrm{s})$ & $\begin{array}{l}\text { Discrepancy }(\% \\
\text { deviation) }\end{array}$ \\
\hline 0.08 & 32.6 & 33.55 & 2.91 \\
0.10 & 25.2 & 23.23 & 7.82 \\
0.12 & 21.6 & 18.46 & 14.54 \\
0.16 & 15.7 & 15.83 & 0.83 \\
\hline
\end{tabular}

\subsection{Validation with Analytical Filling Time Model}

Subsequently, Figure 4 gave another comparison between the filling progressions that obtained from current numerical simulation with that obtained from the regional segregation based analytical filling time model [17-20].

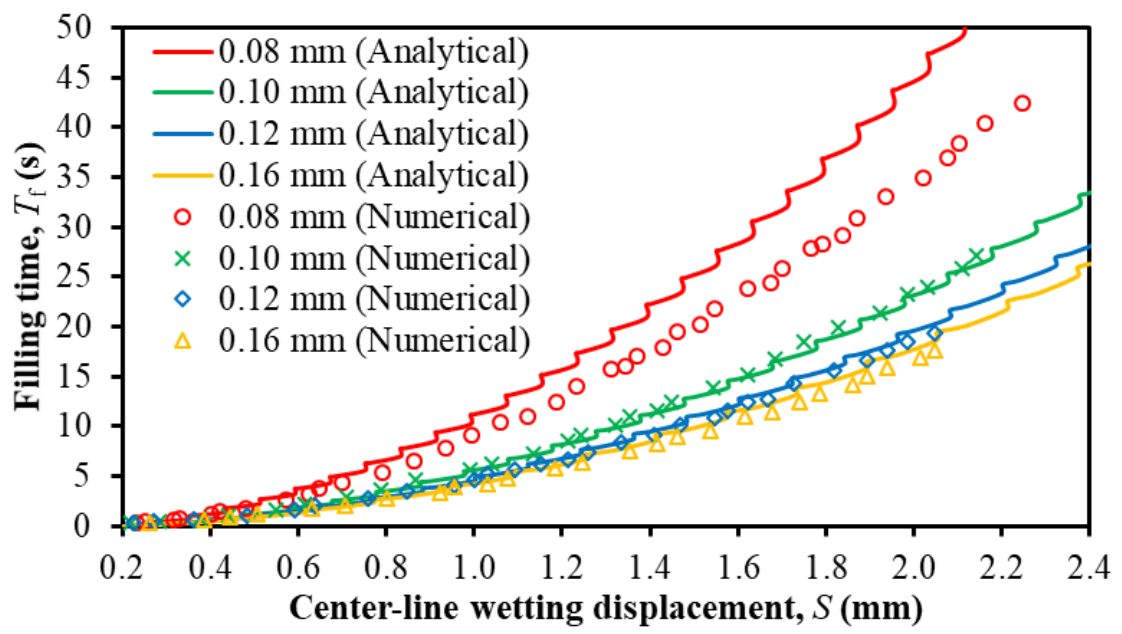

Fig. 4. Comparisons of numerical and analytical filling progressions in the flip-chips with varying pitches

On the general trending, both simulation and analytical models predicted the filling progression as a monotonically increasing curve, that is indicated by the gradually increasing flow of underfill fluid to the flip-chip domain. Both the analytical and numerical findings are in great consensus, where the filling time at any given filling distance is always higher when the bump pitch is shorter. However, the discrepancy between numerical and analytical findings for the case of $0.08 \mathrm{~mm}$ pitch is high, up to $31.41 \%$. The small pitch of $0.08 \mathrm{~mm}$ that close to the pitch threshold gives large magnitude of resisting capillary pressure on the convex meniscus, thus yields a long filling time. For other larger 
pitches, the slight deviation is due to the analytical model did not account the continuous dispensing of underfill fluid as adopted in numerical simulation.

\subsection{Effect of Bump Pitch on Filling Time}

Figure 5 depicts the contours of numerical simulated filling progression of flip-chips of variable pitches. The plot in Figure 6 gives the effect of bump pitch on the filling progression. The filling time at a fixed filling distance increases with the decreases in bump pitch. From Figure 7, the decreasing trend of filling time with the increases of bump pitch becomes more obvious at larger filling distance. The filling time of flow front that locates near to the inlet is almost invariant of the pitch, because of the large pressure gradient of flow. Thus, the impact of bump pitch variation at earlier stage is negligible.

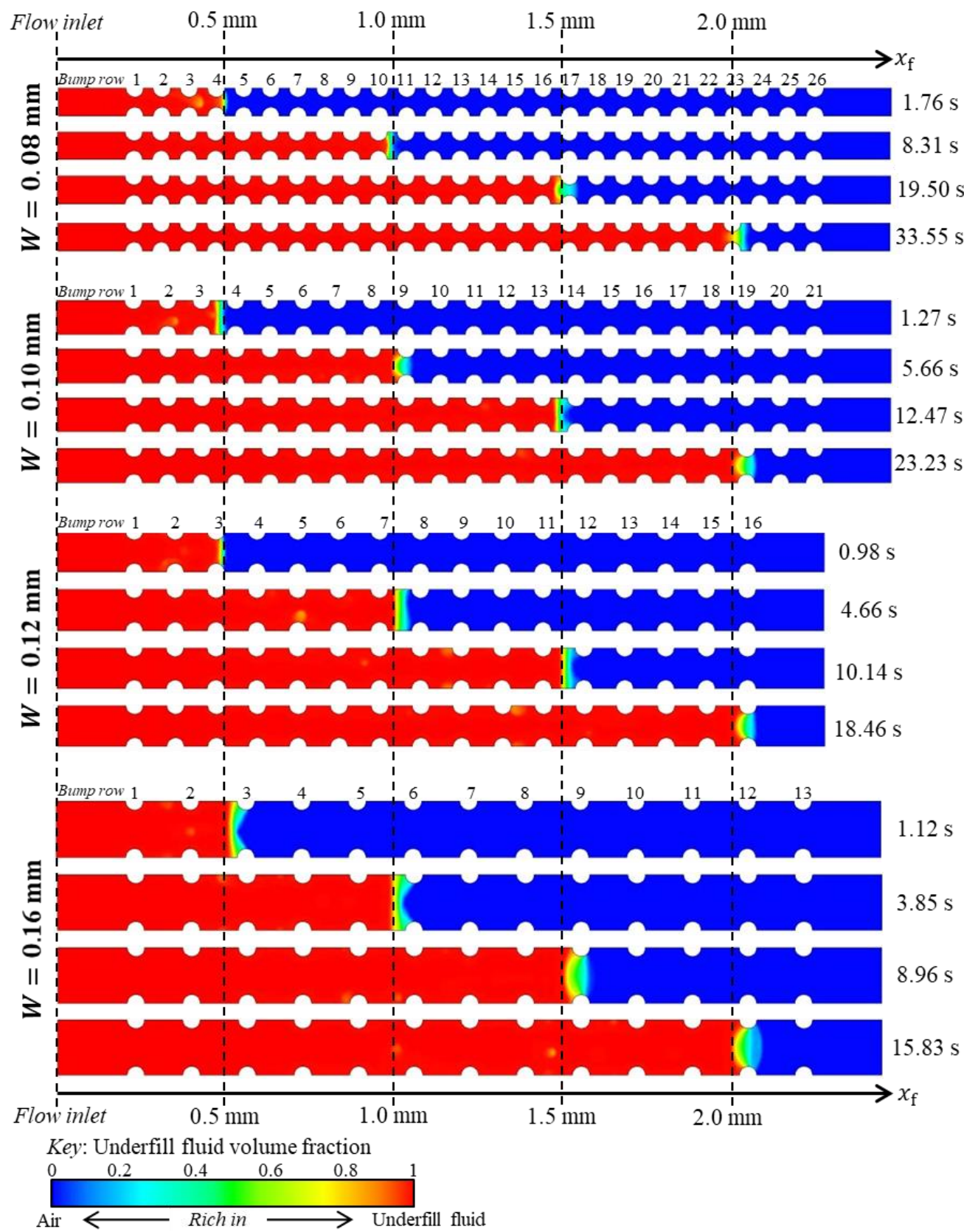

Fig. 5. Numerical flow progression of underfill fluid in the one-pitch width flip-chips of varying pitches 


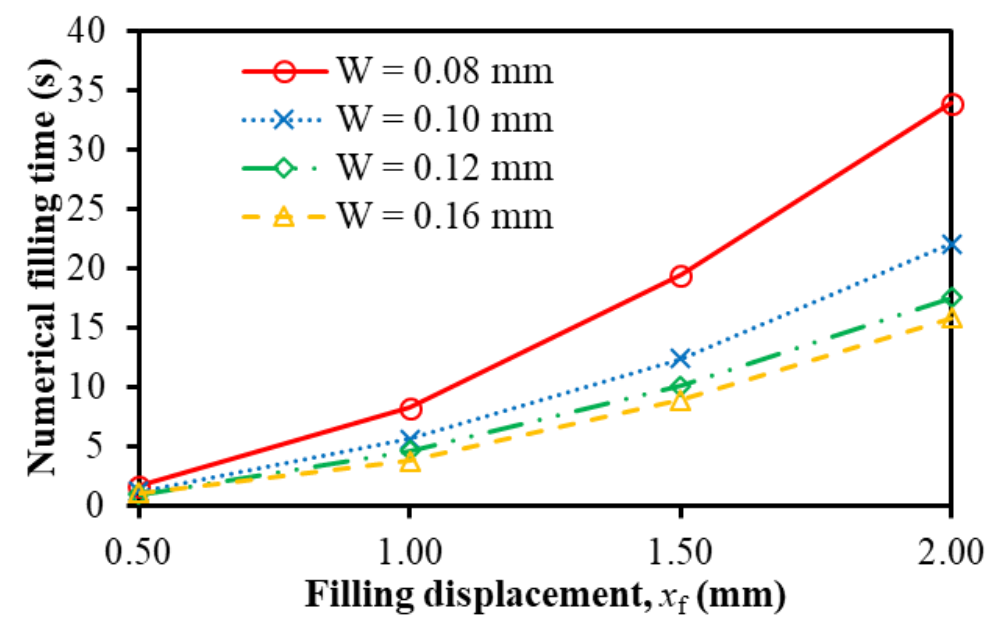

Fig. 6. Numerical filling progression plots for flip-chips with varying bump pitches

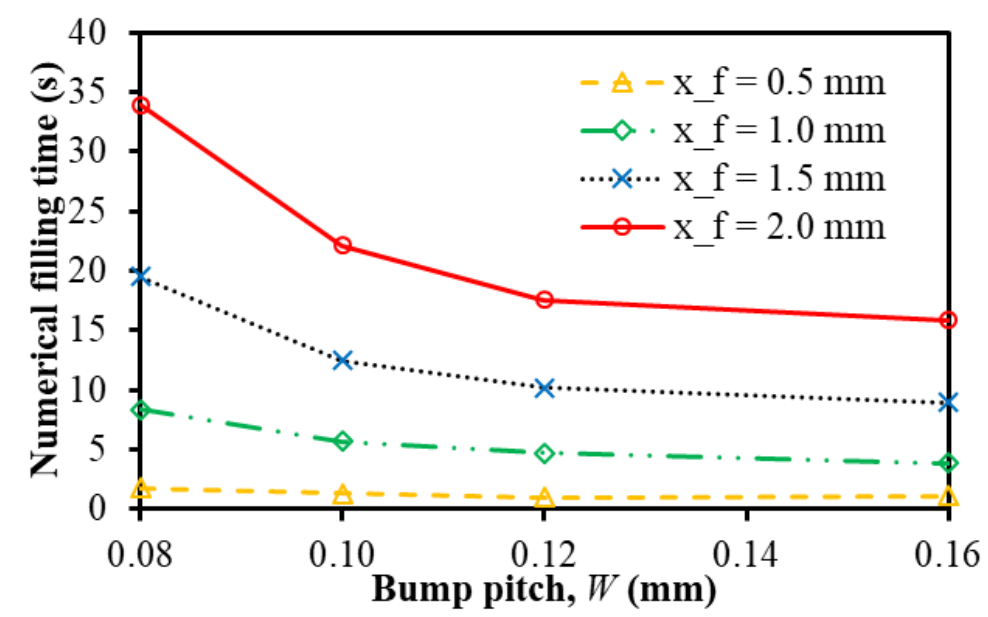

Fig. 7. Plot of numerical filling time for the flow front to reach specific filling distance for various pitch cases

\section{Conclusions}

In this paper, a new symmetrical unit-cells approach using the finite volume method (FVM) based numerical scheme was presented to simulate the underfill fluid flow in bump array of flip-chip during the underfill encapsulation process. Due to repetitive nature of flip-chip bump array, it is sufficient to simulate the underfill flow along the flow direction by considering the one-pitch thick unit-cells array. The walls of cut-plane between two adjacent unit-cells were modelled as periodic boundary condition. Consequently, the current approach greatly reduced the simulation time due to less mesh number on the simplified mathematical model. Moreover, the current numerical findings are wellvalidated with the past underfill experiment and the analytical filling time model. Particularly, the discrepancy between numerical and experimental filling times is not more than $14.54 \%$. Moreover, both analytical and numerical filling progressions are found to in great consensus. By using the proposed numerical approach, the effect of bump pitch on the underfill flow was studied by considering four cases of flip-chips of pitches vary from $0.08 \mathrm{~mm}$ to $0.16 \mathrm{~mm}$. The filling time decreases with the increases in bump pitch; such that the filling time approximately halved when the bump pitch doubled. In sum, the current presented numerical approach is useful for future underfill 
research works, particularly the design optimization study of flip-chip and underfill process, so the underfill simulations can be completed in shorter time without compromising the accuracy.

\section{Acknowledgement}

This research work was partly funded by the Research University (RU) grant (Grant No.: 8014071). Besides, the authors also like to acknowledge the USM Fellowship Award, provided by Institute of Postgraduate Studies (IPS), University Sains Malaysia (USM).

\section{References}

[1] Gnanasegaran, Yogeswaran, Ahmad Anas Yusof, and Mohd Firdaus Shukri. "Study of Thermal-Fluid Analysis on Fusible Metal Bonding Application." Journal of Advanced Research in Fluid Mechanics and Thermal Sciences 62, no. 1 (2019) 88-102.

[2] Ng, Fei Chong, Aizat Abas, Ismail Abustan, Z. Mohd Remy Rozainy, M. Z. Abdullah, and Sharon Melissa Kon. "Visualization of Underfill Flow in Ball Grid Array (BGA) using Particle Image Velocimetry (PIV)." MS\&E 370, no. 1 (2018): 012064. https://doi.org/10.1088/1757-899X/370/1/012064

[3] Ng, Fei Chong, Mohamad Aizat Abas, M. Z. Abdullah, M. H. H. Ishak, and Gean Yuen Chong. "CUF scaling effect on contact angle and threshold pressure." Soldering \& Surface Mount Technology 29 no.4 (2017): 173-190. https://doi.org/10.1088/1757-899X/203/1/012013

[4] Young, Wen-Bin, and Wen-Lin Yang. "The effect of solder bump pitch on the underfill flow." IEEE Transactions on Advanced Packaging 25, no. 4 (2002): 537-542. https://doi.org/10.1109/TADVP.2002.807564

[5] Lee, Seok Hwan, Jaeyong Sung, and Sarah Eunkyung Kim. "Dynamic flow measurements of capillary underfill through a bump array in flip chip package." Microelectronics Reliability 50, no. 12 (2010): 2078-2083. https://doi.org/10.1016/j.microrel.2010.07.001

[6] Ng, Fei Chong, Aizat Abas, and Mohd Zulkifly Abdullah. "Effect of solder bump shapes on underfill flow in flip-chip encapsulation using analytical, numerical and PIV experimental approaches." Microelectronics Reliability 81 (2018): 41-63. https://doi.org/10.1016/i.microrel.2017.12.025

[7] Abas, Aizat, Fei Chong Ng, Z. L. Gan, M. H. H. Ishak, M. Z. Abdullah, and Gean Yuen Chong. "Effect of scale size, orientation type and dispensing method on void formation in the CUF encapsulation of BGA." Sādhanā 43, no. 4 (2018): 59.

https://doi.org/10.1007/s12046-018-0849-3

[8] Ng, Fei Chong, Aizat Abas, Muhammad Hafifi Hafiz Ishak, Mohd Zulkifly Abdullah, and Abdul Aziz. "Effect of thermocapillary action in the underfill encapsulation of multi-stack ball grid array." Microelectronics Reliability 66 (2016): 143-160. https://doi.org/10.1016/j.microrel.2016.10.001

[9] Wang, Jinlin. "The effects of rheological and wetting properties on underfill filler settling and flow voids in flip chip packages." Microelectronics Reliability 47, no. 12 (2007): 1958-1966. https://doi.org/10.1016/j.microrel.2007.04.016

[10] Ng, Fei Chong, Aizat Abas, Z. L. Gan, Mohd Zulkifly Abdullah, F. Che Ani, and M. Yusuf Tura Ali. "Discrete phase method study of ball grid array underfill process using nano-silica filler-reinforced composite-encapsulant with varying filler loadings." Microelectronics Reliability 72 (2017): 45-64. https://doi.org/10.1016/i.microrel.2017.03.034

[11] Han, Sejin, and K. K. Wang. "Analysis of the flow of encapsulant during underfill encapsulation of flip-chips." IEEE Transactions on Components, Packaging, and Manufacturing Technology: Part B 20, no. 4 (1997): 424-433. https://doi.org/10.1109/96.641511

[12] Wan, J. W., W. J. Zhang, and D. J. Bergstrom. "Numerical modeling for the underfill flow in flip-chip packaging." IEEE Transactions on Components and Packaging Technologies 32, no. 2 (2009): 227-234. https://doi.org/10.1109/TCAPT.2009.2014355

[13] Ng, F. C., A. Abas, and M. Z. Abdullah. "Finite volume method study on contact line jump phenomena and dynamic contact angle of underfill flow in flip-chip of various bump pitches." In IOP Conference Series: Materials Science and Engineering, vol. 530, no. 1, p. 012012. IOP Publishing, 2019. https://doi.org/10.1088/1757-899X/530/1/012012 
[14] Kulkarni, Venkatesh M., K. N. Seetharmu, PA Aswatha Narayana, I. A. Azid, and G. A. Quadir. "Flow analysis for flip chip underfilling process using characteristic based split method." In Proceedings of 6th Electronics Packaging Technology Conference (EPTC 2004)(IEEE Cat. No. 04EX971), pp. 615-619. IEEE, 2004. https://doi.org/10.1109/EPTC.2004.1396681

[15] Wang, Hui, Huamin Zhou, Yun Zhang, Dequn Li, and Kai Xu. "Three-dimensional simulation of underfill process in flip-chip encapsulation." Computers \& fluids 44, no. 1 (2011): 187-201. https://doi.org/10.1016/i.compfluid.2010.12.030

[16] Yao, Xing Jun, Zhengdong Wang, Wenjun Zhang, and Xingyan Zhou. "A new model for permeability of porous medium in the case of flip-chip packaging." IEEE Transactions on Components, Packaging and Manufacturing Technology 4, no. 8 (2014): 1265-1275. https://doi.org/10.1109/TCPMT.2014.2316537

[17] Ng, Fei Chong, Aizat Abas, and M. Z. Abdullah. "Regional Segregation With Spatial Considerations-Based Analytical Filling Time Modelfor Non-Newtonian Power-Law Underfill Fluid in Flip-Chip Encapsulation." Journal of Electronic Packaging 141, no. 4 (2019). https://doi.org/10.1115/1.4044817

[18] Ng, Fei Chong, Mohamad Aizat Abas, and Mohd Zulkifly Abdullah. "Filling efficiency of flip-chip underfill encapsulation process." Soldering \& Surface Mount Technology 32 no. 1 (2019):10-18. https://doi.org/10.1108/SSMT-07-2019-0026

[19] Ng, Fei Chong, M. Yusuf Tura Ali, Aizat Abas, C. Y. Khor, Z. Samsudin, and M. Z. Abdullah. "A novel analytical filling time chart for design optimization of flip-chip underfill encapsulation process." The International Journal of Advanced Manufacturing Technology 105, no. 7-8 (2019): 3521-3530. https://doi.org/10.1007/s00170-019-04573-6

[20] Ng, Fei Chong, Lun Hao Tung, Mohd Hafiz Zawawi, Muhamed Abdul Fatah Muhamed Mukhtar, Mohamad Aizat Abas, and Mohd Zulkifly Abdullah. "Effect of Contact Angle on Meniscus Evolution and Contact Line Jump of Underfill Fluid Flow in Flip-Chip Encapsulation." CFD Letters 12, no. 6 (2020): 28-38.

https://doi.org/10.37934/cfdl.12.6.2838 